Pacific Journal of Mathematics

THE SLOW STEADY MOTION OF LIQUID PAST A 


\title{
THE SLOW STEADY MOTION OF LIQUID \\ PAST A SEMI-ELLIPTICAL BOSS
}

\author{
G. Power and D. L. Scott-Hutton
}

1. Introduction. In this problem of two-dimensional viscous flow, liquid is supposed to have a rigid boundary represented by $A B C D E$ in Figure 1 and, apart from the disturbance caused by the presence of the elliptical boss $B C D$, is assumed to be in uniform shearing motion. The stream function is thus a biharmonic function vanishing together with its normal derivative at all points of the boundary, and proportional to $y^{2}$ at a great distance from the boss. A series of functions is found, each of which satisfies all the boundary conditions save one. A linear combination of these functions will also satisfy the boundary conditions with this one exception, and by a particular choice of the arbitrary constants which it contains, the remaining condition can be satisfied at as many points as desired. Special cases are discussed, and a process of approximation is outlined which yields the most accurate results at $C$, and also gives a convenient function for determining at any point of the boundary the magnitude of the error in the unsatisfied boundary condition. A special case of this problem has previously been considered [1].

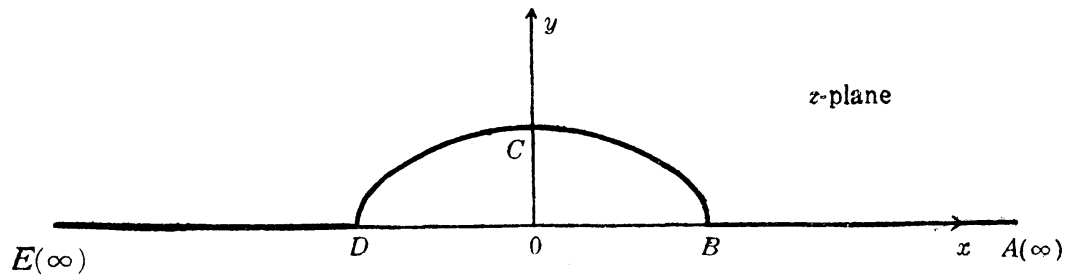

Figure 1.

2. The stream function. We take the equation of the boundary $B C D$ to be $x^{2} / a^{2}+y^{2} / b^{2}=1$, and note that the region occupied by the fluid, for which $y$ is never negative, is transformed into the interior of the semi-circle of unit radius shown in Figure 2 by

$$
-2 z=(a-b) w+(a+b) / w .
$$

The stream function $\psi$ is biharmonic, that is to say it must satisfy $\nabla^{ \pm} \psi=0$, and a satisfactory solution to the problem is

$$
\phi=y^{2}+U+y V
$$

Received September 14, 1954. 


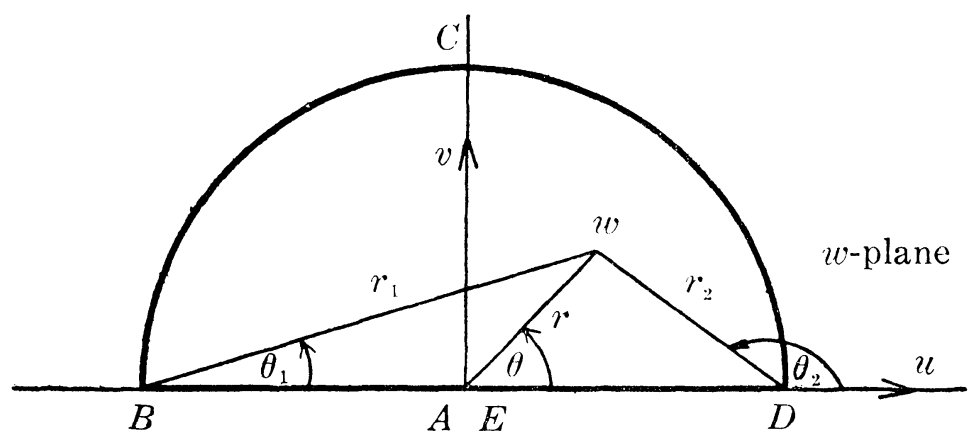

Figure 2.

provided $U$ and $V$ are harmonic functions which are chosen so that $U+y V$ does not tends to infinity as $z$ tends to infinity.

The boundary conditions to be satisfied are $\psi=0$ and $\partial \psi / \partial \nu=0$ along the boundary, where $\delta \nu$ denotes an element of normal to the boundary. From (2), we see that four conditions are required, namely
(a) $U=0$
along $A B$ and $D E$, that is when $y=0$,
(b) $V+\partial U / \partial y=0$
along $A B$ and $D E$, that is when $y=0$,
(c) $y^{2}+U+y V=0$
along $B C D$, that is when $x^{2} / a^{2}+y^{2} / b^{2}=1$,
(d) $(\partial / \partial \nu)\left(y^{2}+U+y V\right)=0$ along $B C D$, that is when $x^{2} / a^{2}+y^{2} / b^{2}=1$.

Writing $w=r e^{i \theta}=u+i v$, and using the tranformation (1), we see that the boundary conditions (3) become, after a little reduction,

(a) $U=0$, when $v=0$,

(b) $(b-a) V^{\prime}+\frac{2 u^{2}}{\left(c+u^{2}\right)} \cdot \frac{\partial U}{\partial v}=0$, when $v=0$,

(c) $U+b \sin \theta V^{\prime}=0$, when $r=1$,

(d) $\quad b(a+b) \sin \theta=\frac{1}{\sin \theta} \partial r+b \frac{\partial V^{\prime}}{\partial r}-a V^{\prime}$, when $r=1$, where $V^{\prime}=V+b r \sin \theta$, and $c=(b+a) /(b-a)$.

We will proceed to find pairs of harmonic functions $U_{2 n-1}, V_{: n-1}^{\prime}$, such that each pair will satisfy exactly the first three of the above boundary conditions. Any linear combination of these functions will also satisfy exactly these three conditions, and by giving special values to the arbitrary constants in this linear combination we can satisfy approximately the fourth equation. Physically, this means that in the fluid motion represented by our solution there will be a small velocity of slip along the boss $B C D$, which can be calculated from the error involved in the last boundary condition. 
If we take

$$
U=\sum_{n=1}^{q} a_{2 n-1} U_{2 n-1}
$$

where

$$
U_{2 n-1}=\mathscr{Y}\left\{\frac{A_{2 n-1}}{2 n+1} w^{2 n+1}+\frac{B_{2 n-1}}{2 n-1} w^{2 n-1}+\frac{C_{2 n-1}}{2 n-3} w^{2 n-3}\right\}
$$

then $U$ is harmonic, and 4 (a) is satisfied. Moreover, the consideration of symmetry shows that even powers of $w$ are not required. Now we have

$$
\begin{aligned}
\left(\frac{\partial U_{2 n-1}}{\partial v}\right)_{v=0}=\left(u^{2}+c\right)\left(A_{2 n-1} u^{2 n-2}+\frac{C_{2 n-1}}{c} u^{2 n-1}\right), & \\
& \text { provided } c A_{2 n-1}-B_{2 n-1}+C_{2 n-1} / c=0,
\end{aligned}
$$

and

$$
\begin{aligned}
\left(U_{2 n-1}\right)_{r=1}=\sin \theta\left(\frac{2 A_{2 n-1}}{2 n+1} \cos 2 n \theta-\frac{2 C_{2 n-1}}{2 n-3} \cos (2 n-2) \theta\right) & \\
& \text { provided } \frac{A_{2 n-1}}{2 n+1}+\frac{B_{2 n-1}}{2 n-1}+\frac{C_{2 n-1}}{2 n-3}=0 .
\end{aligned}
$$

From (7), (8), we see that we can take

$$
\begin{aligned}
& A_{2 n-1}=\frac{2(2 n+1)}{b-a}\{2(n-1) b+a\} p_{n}, \\
& B_{2 n-1}=\frac{4(2 n-1)}{(b-a)^{2}}\left\{b^{2}+(2 n-1) a b+a^{2}\right\} p_{n}, \\
& C_{2 n-1}=-\frac{2(2 n-3)(b+a)}{(b-a)^{2}}\{2 n b+a\} p_{n},
\end{aligned}
$$

where the unknown $p_{n}$ has yet to be determined. By setting

$$
V_{2 n-1}^{\prime}=W_{2 n-1}+\frac{2 C_{2 n-1} r^{2 n-2} \cos (2 n-2) \theta}{(2 n-3) b}-\frac{2 A_{2 n-1} r^{2 n} \cos 2 n \theta}{(2 n+1) b}
$$

we see that $4(c)$ yields

$$
W_{2 n-1}=0 \quad \text { when } \quad r=1 .
$$

If further we take

$$
p_{n}=\frac{b(b-a)^{2}}{4(2 n b+a)\{2(n-1) b+a\}}
$$


then condition $4(\mathrm{~b})$ gives

$$
W_{2 n-1}=u^{2 n-2}-u^{n n} \quad \text { when } \quad v=0 .
$$

Equations (9), (11) now give

$$
\begin{aligned}
& A_{2 n-1}=\frac{(2 n+1) b(b-a)}{2(2 n b+a)}, \\
& B_{2 n-1}=\begin{array}{c}
(2 n-1) b\left\{b^{2}+(2 n-1) a b+a^{2}\right\} \\
(2 n b+a)\{2(n-1) b+a\}
\end{array}, \\
& C_{2 n-1}=-\begin{array}{r}
(2 n-3) b(b+a) \\
2\{2(n-1) b+a\}
\end{array} .
\end{aligned}
$$

To find the function $W_{2 n-1}$ satisfying (10), (12), we consider $\psi_{2 n}=\mathscr{R}\left\{\chi_{2 n}(w)\right\}$, where

$$
\left\{\begin{array}{l}
\chi_{2 n}(w)=-w^{-2 n}+\frac{i}{\pi}\left\{w^{2 n}+\frac{1}{w^{2 n}}\right\}\{\log (w+1)-\log (w-1)\} \\
-\frac{2 i}{\pi}\left\{\left(w^{2 n-1}+\frac{1}{w^{2 n-1}}\right)+\frac{1}{3}\left(w^{2 n-3}+\frac{1}{w^{2 n-3}}\right)+\cdots+\frac{1}{2 n-1}\left(w+\frac{1}{w}\right)\right\}, \\
\chi_{0}(w)=-1+\frac{2 i}{\pi}\{\log (w+1)-\log (w-1)\} .
\end{array}\right.
$$

It is easy to verify that $\mathscr{R}\left\{\chi_{2 n}(w)\right\}=0$ when $r=1$, and that $\mathscr{R}\left\{\chi_{\Perp_{n}}(w)\right\}=u^{\sharp n}$ when $v=0$, since from Figure 2

$$
\log (w-1)-\log (w+1)=\log \frac{r_{2}}{r_{1}}+i\left(\theta_{2}-\theta_{1}\right) .
$$

The function $W_{2 n-1}$ is thus given by

$$
W_{2 n-1}=\phi_{2 n-2}-\phi_{2 n} .
$$

Finally, we see that the required stream function $\psi$ is given by equation (2), where

$$
\begin{gathered}
U=\mathscr{F} \sum_{n=1}^{q} a_{2 n-1}\left\{\begin{array}{c}
A_{2 n-1} \\
2 n+1
\end{array} w^{2 n+1}+\begin{array}{c}
B_{2 n-1} \\
2 n-1
\end{array} w^{n n-1}+\begin{array}{r}
C_{2 n-1} \\
2 n-3
\end{array} w^{2 n-3}\right\}, \\
V=\mathscr{R}\left\{i b w+\sum_{n=1}^{q} a_{2 n-1}\left\{\begin{array}{c}
2 C_{2 n-1} \\
(2 n-3) b
\end{array} w^{2 n-2}-\begin{array}{c}
2 A_{2 n-1} \\
(2 n+1) b
\end{array} w^{2 n}\right.\right. \\
\left.\left.+\chi_{2 n-2}(w)-\chi_{2 n}(w)\right\}\right\},
\end{gathered}
$$

the constants $A_{2 n-1}, B_{2 n-1}, C_{2 n-1}$ being determined from (13), $\chi_{2 n}(w)$ from (14). It is quite easy to verify that $\psi \rightarrow y^{2}$ as $z \rightarrow \infty$, that is as $w \rightarrow 0$, since the most significant terms in $U$ and $y V$ are respectively 
$2 a_{1} C_{1} y /(a+b)$ and $a_{1} y\left(-2 C_{1} / b+1\right)$, the sum of these being clearly zero from (13).

3. The fourth boundary condition. It is now necessary to consider the boundary condition as yet unsatisfied, given by equation 4 (d), in the form

$$
b(a+b) \sin \theta=\sum_{n=1}^{q} a_{2 n-1} \lambda_{2 n-1}(\theta) \quad \text { when } r=1,
$$

where

$$
\lambda_{2 n-1}(\theta)=\frac{1}{\sin \theta} \frac{\partial U_{2 n-1}}{\partial r^{-}}+b^{\partial V_{2 n-1}^{\prime}} \frac{a V_{2 n-1}^{\prime}}{\partial r^{-}} .
$$

Theoretically, the constants $a_{2 n-1}$ must be chosen so that (17) is satisfied for all values of $\theta$ lying between 0 and $\pi$, and this would require an infinite number of terms. Clearly, therefore, some form of approximation must be applied. Suppose that the constants $a_{2 n-1}$ are chosen so that

$$
\sum_{n=1}^{q} a_{2 n-1} \lambda_{2 n-1}=b(a+b) \sin \theta+F(\theta)
$$

then $\sin \theta F(\theta)$ is the error involved in the boundary derivative $(\partial \psi / \partial r)_{r=1}$, and the actual velocity of slip on the boundary $B C D$ in the $z$-plane is

$$
\left\{\left(\begin{array}{c}
\partial \psi \\
\partial r
\end{array}\right)\left|\begin{array}{c}
d w \\
d z
\end{array}\right|\right\}_{r=1}=\frac{\sin \theta F(\theta)}{\left(b^{2} \cos ^{2} \theta+a^{2} \sin ^{2} \theta\right)^{1 / 2}}
$$

This becomes infinite at $\theta=\pi / 2$ in the degenerate case $a=0$, unless $F(\pi / 2)$ happens to be zero. Therefore we must consider a method of approximation which gives no error at all when $\theta=\pi / 2$. The coefficients $a_{2 n-1}$ will be chosen so that the expressions on each side of (17) have the same values and the same differential coefficients with regard to $\theta$ when $\theta=\pi / 2$, the number of differential coefficients that can be equated depending on the value of the integer $q$. From (16), we see that when $r=1$,

$$
\text { (18) } \begin{aligned}
\lambda_{2 n-1}(\theta) & =\frac{1}{\sin \theta}\left\{A_{2 n-1} \sin (2 n+1) \theta+B_{2 n-1} \sin (2 n-1) \theta+C_{2 n-1} \sin (2 n-3) \theta\right\} \\
& +2\left\{\frac{2(n-1) b-a}{(2 n-3) b}\right\} C_{2 n-1} \cos (2 n-2) \theta-2\left\{\begin{array}{c}
2 n b-a \\
(2 n+1) b
\end{array}\right\} A_{2 n-1} \cos 2 n \theta \\
& +b\left(f_{2 n-2}(\theta)-f_{2 n}(\theta)\right),
\end{aligned}
$$

where 


$$
\begin{aligned}
f_{2 n}(\theta)= & \left(\frac{\partial \phi_{2 n}}{\partial r}\right)_{r=1}=\Re\left\{\begin{array}{c}
d \chi_{2 n}(w) \\
d w
\end{array}\right\}_{r=1} \\
= & \frac{8 n}{\pi}\left\{\sin (2 n-1) \theta+\frac{1}{3} \sin (2 n-3) \theta+\cdots+\frac{1}{2 n-1} \sin \theta\right\} \\
& +2 n \cos 2 n \theta+\frac{4 n}{\pi} \sin 2 n \theta \log \tan \frac{\theta}{2}-\frac{2}{\pi} \csc \theta, \\
f_{0}(\theta)= & -\frac{2}{\pi} \csc \theta .
\end{aligned}
$$

It is to be noted, that although $f_{2 n}(\theta)$ is infinite at $\theta=0, \pi$, the expression $f_{2 n-2}(\theta)-f_{2 n}(\theta)$, which occurs in $\lambda_{2 n-1}(\theta)$ is finite at these points. Equation (17) is satisfied exactly when $\theta=0, \pi$, and putting $\theta=\pi / 2$, we have

$$
b(a+b)=\sum_{n=1}^{q} a_{2 n-1} \lambda_{2 n-1}(\pi / 2),
$$

and by differentiation we are led to

$$
\left\{\begin{aligned}
-b(a+b) & =\sum_{n=1}^{q} a_{2 n-1} \lambda_{2 n-1}^{\prime \prime}(\pi / 2), \\
b(a+b) & =\sum_{n=1}^{q} a_{2 n-1} \lambda_{2 n-1}^{l V}(\pi / 2),
\end{aligned}\right.
$$

and so forth.

It is from this set of equations that the constants $a_{2 n-1}$ are to be calculated.

4. Special cases. The two special cases of the semi-circular boss and projection will now be discussed.

$(\alpha)$ semi-circular boss.

Setting $a=b=1$, equation (16) yields

$$
\begin{aligned}
& U=\sum_{n=1}^{q} \frac{a_{2 n-1}}{2 n-1}\left\{r^{2 n-1} \sin (2 n-1) \theta-r^{2 n-3} \sin (2 n-3) \theta\right\}, \\
& V=-r \sin \theta+\sum_{n=1}^{q} a_{2 n-1}\left(\phi_{2 n-2}-\phi_{2 n}\right)-\sum_{n=1}^{q} 2 a_{2 n-1} r^{2 n-2} \cos (2 n-2) \theta,
\end{aligned}
$$

and from (18), we get when $r=1$

$$
\lambda_{2 n-1}(\theta)=\frac{1}{\sin \theta}\left\{\begin{array}{c}
2 \\
2 n-1
\end{array} \sin (2 n-1) \theta\right\}+f_{2 n-2}(\theta)-f_{2 n}(\theta) .
$$

As an example, let us take $q=3$, so that from (19) we are required to solve the equations 


$$
\begin{aligned}
2 & =a_{1} \lambda_{1}(\pi / 2)+a_{3} \lambda_{3}(\pi / 2)+a_{5} \lambda_{5}(\pi / 2), \\
-2 & =a_{1} \lambda_{1}^{\prime \prime}(\pi / 2)+a_{3} \lambda_{3}^{\prime \prime}(\pi / 2)+a_{5} \lambda_{5}^{\prime \prime}(\pi / 2), \\
2 & =a_{1} \lambda_{1}^{I V}(\pi / 2)+a_{3} \lambda_{3}^{I V}(\pi / 2)+a_{5} \lambda_{5}^{I V}(\pi / 2) .
\end{aligned}
$$

By substitution and straightforward calculation we obtain the following table :-

\begin{tabular}{c|ccc} 
& $n=1$ & $n=2$ & $n=3$ \\
\hline$\lambda_{2 n-1}(\pi / 2)$ & +1.45352 & -0.72488 & +0.38385 \\
$\lambda_{2 n-1}^{\prime \prime}(\pi / 2)$ & -0.36056 & +5.18309 & -9.65706 \\
$\lambda_{2 n-2}^{I ! r}(\pi / 2)$ & -1.10423 & -22.24210 & +191.53800
\end{tabular}

which leads directly to $a_{1}=+1.21058, a_{3}=-0.34379, a_{5}=-0.02299$.

A more accurate result can be obtained by taking more terms in the linear expression for $\psi$, and it is found that the coefficients $a_{2 n-1}$ decrease rapidly in magnitude, but the numerical work involved soon becomes exceedingly heavy.

This choice of approximation method is seen to advantage if calculating the error function

$$
F(\theta)=\sum_{n=1}^{3} a_{2 n-1} \lambda_{2 n-1}(\theta)-2 \sin \theta
$$

at any point by means of the Taylor expansion about $\theta=\pi / 2$, several of the significant differential coefficients being zero by definition. The following table gives the value of $F(\theta)$ for various values of $\theta$, and Figure 3 shows the graph of $F(\theta)$ plotted against values of $\theta$ lying between 0 and $\pi / 2$. The graph for $\pi / 2 \leq \theta \leq \pi$ will, of course, be similar, since $F(\theta)$ is symmetrical about $\theta=\pi / 2$.

\begin{tabular}{|c|c|cc|c|c|cc|}
\hline$\theta$ & 0 & $\pi / 45$ & $\pi / 15$ & $\pi / 8$ & $\pi / 4$ & $3 \pi / 8$ & $\pi / 2$ \\
\hline $\boldsymbol{F}(\theta)$ & 0 & 0.05918 & 0.08225 & 0.05838 & 0.00915 & 0.00041 & 0 \\
\hline
\end{tabular}

It will be noticed that the results are most accurate in the vicinity of $\theta=\pi / 2$, as might be expected from the method of approximation. Although the value of $F(\theta)$ becomes greater than 0.08 for a certain (small) values of $\theta$, the velocity of slip, given by $\sin \theta \cdot F(\theta)$, is really very small at these points.

( $\beta)$ degenerate boss.

If $a=0$, the semi-elliptical boss degenerates to a projection into 


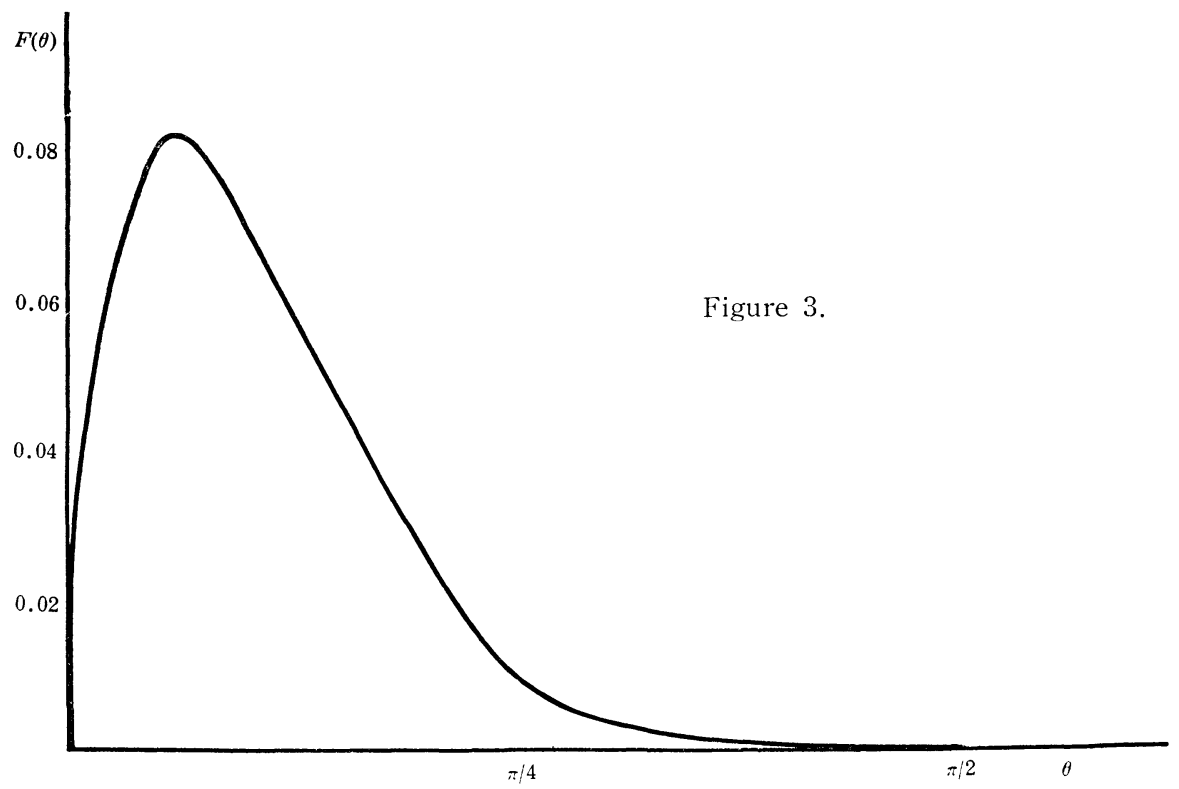

the moving liquid, and the formulae (13) become

$$
A_{2 n-1}=\frac{(2 n+1) b}{4 n}, \quad B_{2 n-1}=\begin{gathered}
(2 n-1) b \\
4 n(n-1)
\end{gathered}, \quad C_{2 n-1}=-\begin{gathered}
(2 n-3) b \\
4(n-1)
\end{gathered} .
$$

These values will hold except for $n=1$. For this case we will follow W. R. Dean [1], and will find a pair of solutions $U_{1}, V_{1}^{\prime}$ which satisfy equations 4 (a), (b), (c). The procedure outlined in $\S 2$ is again followed, and omitting details we are led to the two pairs of solutions

$$
U_{1}=\mathscr{\gamma} b w, \quad V_{1}^{\prime}=-\mathscr{R}\left\{\begin{array}{c}
2 w \\
i+w
\end{array}\right\},
$$

and

$$
\mathscr{F}_{4}^{b}\left\{w^{3}+3 w\right\}, \quad U_{1}=\frac{w^{3}}{4}+w, \quad V_{1}^{\prime}=\mathscr{P}\left\{-1-\frac{1}{2} w^{2}+\chi_{0}(w)-\chi_{2}(w)\right\} .
$$

The final solution is thus

$$
\left\{\begin{aligned}
U= & \mathscr{F}\left\{a_{0} b w+\frac{a_{1} b}{4}\left(w^{3}+3 w\right)+\sum_{n=2}^{q} a_{2 n-1} b\left(\begin{array}{c}
w^{2 n+1}+\frac{w^{2 n-1}}{4 n}+w^{2 n-3} \\
4 n(n-1)
\end{array}\right)\right\} \\
V= & \left.\mathscr{R}\left\{i b w+a_{1}\left(-1-\frac{1}{2} w^{2}\right)+\sum_{n=2}^{q} a_{2 n-1}\left(\begin{array}{c}
w^{2 n-2} \\
2(n-1)
\end{array}\right)+\begin{array}{c}
w^{2 n} \\
2 n
\end{array}\right)\right\} \\
& +\mathscr{R}\left\{-\begin{array}{l}
2 a_{0} w \\
i+w
\end{array}+\sum_{n=1}^{q} a_{2 n-1}\left(\chi_{2 n-2}(w)-\chi_{2 n}(w)\right)\right\} .
\end{aligned}\right.
$$


Again we note that $\psi \rightarrow y^{2}$ as $z \rightarrow \infty$.

5. The pressure equation. The pressure $p$ is determined from the equations of motion in the form

$$
\frac{\partial p}{\partial x}=-\mu \frac{\partial}{\partial y} \nabla^{2} \psi, \quad \frac{\partial p}{\partial y}=\mu \frac{\partial}{\partial x} \nabla^{2} \psi^{\prime},
$$

where $\mu$ is the coefficient of viscosity. Now $\psi=y^{2}+U+y V$, where $U$ and $V$ are harmonic, so that $\nabla^{\prime \prime} \psi=2+2(\partial V / \partial y)$, and hence

$$
\frac{\partial p}{\partial x}=-2 \mu \frac{\partial^{2} V}{\partial y^{2}}=2 \mu \begin{gathered}
\partial^{2} V \\
\partial x^{2}
\end{gathered}, \quad \frac{\partial p}{\partial y}=2 \mu \partial^{2} V .
$$

Ignoring an arbitrary constant, we have therefore

$$
p=2 \mu \frac{\partial V}{\partial x}=2 \mu \mathscr{R}\left\{\begin{array}{c}
d V(w) \\
d z
\end{array}\right\}=2 \mu \mathscr{R}\left\{\frac{d w}{d z} \cdot \frac{d V(w)}{d w}\right\},
$$

where $V=\mathscr{R}\{V(w)\}$. From equation (16) we see that

$$
V(w)=i b w+\sum_{n=1}^{q} a_{2 n-1}\left\{\frac{2 C_{2 n-1} w^{2 n-2}}{(2 n-3) b}-\frac{2 A_{2 n-1} w^{2 n}}{(2 n+1) b}+\chi_{2 n-2}(w)-\chi_{2 n}(w)\right\},
$$

and so

$$
\begin{aligned}
& p=2 \mu \mathscr{R}\left\{[ \begin{array} { c } 
{ 2 w ^ { 2 } } \\
{ ( b + a ) + ( b - a ) w ^ { 2 } }
\end{array} ] \left[i b+\sum_{n=1}^{q} a_{2 n-1}\left\{\frac{4(n-1) C_{2 n-1} w^{2 n-3}}{(2 n-3) b}\right.\right.\right.
\end{aligned}
$$

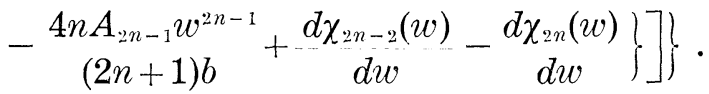

Equation (21) gives the pressure distribution, and on the plane boundary, where $v=0$, this becomes

$$
\begin{aligned}
p=\left\{\frac{4 \mu u^{2}}{(b+a)+(b-a) u^{2}}\right\} & \left\{\sum _ { n = 1 } ^ { q } a _ { 2 n - 1 } \left\{\begin{array}{c}
4(n-1) C_{2 n-1} u^{2 n-3} \\
(2 n-3) b
\end{array}\right.\right. \\
- & \left.\begin{array}{c}
\left.4 n A_{2 n-1} u^{2 n-1}+2(n-1) u^{2 n-3}-2 n \iota^{2 n-1}\right\} \\
(2 n+1) b
\end{array}\right\} .
\end{aligned}
$$

In particular the pressure at $B$ exceeds that at $D$ by

$$
p_{\text {difr }}=\frac{8 \mu}{b} \sum_{n=1}^{q} a_{2 n-1}\left\{-\frac{2(n-1) C_{2 n-1}}{(2 n-3) b}+\frac{2 n A_{2 n-1}}{(2 n+1) b}+1\right\} .
$$

For the special case $a=b=1$ discussed in $\S 4(\alpha)$, this expression is

$$
8 \mu \sum_{n=1}^{q} \frac{(4 n-3) a_{2 n-1}}{2 n-1}
$$


and with the values for $a_{1}, a_{3}, a_{5}$ substituted we obtain a difference of $4.78 \mu$.

For the degenerate case $a=0, b=1$, Dean [1] obtains a difference of approximately $5.80 \mu$ between the pressures at $B$ and $D$.

\section{REFERENCES}

1. W. R. Dean, Note on the slow motion of fluid. Proc. Cambridge Philos. Soc., 32 (1936), 598.

The University of NotTingham

Added in Proof: The equations governing the slow steady flow of a viscous incompressible fluid are the same as those characterizing an equilibrium state of an incompressible elastic solid, if one simply replaces velocity and coefficient of viscosity by displacement and shear modulus. Thus the results here obtained can be used as the solution for the tension of a semi-infinite plane whose edge is indented and traction free. 
.././. ./FrontMatter/paper .pdf 


\section{Pacific Journal of Mathematics}

Nesmith Cornett Ankeny and Theodore Joseph Rivlin, On a theorem of S.

Bernstei........................................ 849

Louis Auslander, The use of forms in variational calculation .......... 853

Paul Civin, Abstract Riemann sum . .......................... 861

Paul Civin, Some ergodic theorems involving two operator ............ 869

Eckford Cohen, The number of solutions of certain cubic congruence .... . 877

Richard M. Cohn, Specializations over difference field .............. 887

Jean Dieudonné, Pseudo-discriminant and Dickson invarian . . ......... 907

Ky Fan, A comparison theorem for eigenvalues of normal matrice ........ 911

Richard P. Gosselin, On the convergence behaviour of trigonometric interpolating polynomial ........................... 915

Peter K. Henrici, On generating functions of the Jacobi polynomial . . . . . . . 923

Meyer Jerison, An algebra associated with a compact grou ............ 933

Wilhelm Magnus, Infinite determinants associated with Hill's equatio . . . . . 941

G. Power and D. L. Scott-Hutton, The slow steady motion of liquid past a semi-elliptical bos................................. 953

Lyle E. Pursell, An algebraic characterization of fixed ideals in certain function ring .................................... 963

C. T. Rajagopal, Additional note on some Tauberian theorems of O. Szás . . 971 Louis Baker Rall, Error bounds for iterative solutions of Fredholm integral

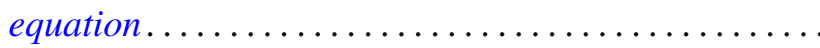

Shigeo Sasaki and Kentaro Yano, Pseudo-analytic vectors on

pseudo-Kählerian manifold ......................

Eugene Schenkman, On the tower theorem for finite group

P. Stein and John E. L. Peck, On the numerical solution of Poisson's equation over a rectangl ........................

Morgan Ward, The mappings of the positive integers into themselves which preserve divisio .

Seth Warner, Weak locally multiplicatively-convex algebra 1025

Louis Weisner, Group-theoretic origin of certain generating function .... 